\title{
26 Construction of personal meaning through the use of advanced geometrical software: the case of trigonometry
}

\author{
Morten Blomhøj \\ IMFUFA, Roskilde University \\ Roskilde, Denmark
}

\begin{abstract}
Analyses of classroom observations of an episode in a special electronic mathematics class in the Danish Upper Secondary School (pupils aged 16-19)all pupils have their own PC and two pupils are working with the law of sinesindicates how the pupils create personal meaning through their use of the Geometer's Sketchpad Program (GSP). The degree of openness of the task and the challenging dialogue conducted with the teacher are seen to be decisive within the activity, but not without considerable demand on the teacher in terms of the teacher's knowledge and ability in supporting the pupils' in their acquisition of the desired outcomes.
\end{abstract}

\section{Keywords}

Classroom practice, learner centred learning, trigonometry.

\section{Construction of episodes as a method for analysing computer-based learning}

The research method I have utilised in my investigation of the significance of computers for teaching and learning mathematics may be characterised as didactic analysis of construed episodes of mathematics teaching (Blomhøj, 1997). My observations of mathematics teaching in the electronic classes, in this case pupils using the Geometer's Sketchpad Program (GSP), where I have had the role of 'participant observer', have provided inspiration for constructing a series of teaching episodes where the use of computers plays a decisive role in pupil activity.

An episode: the law of sines saturated with personal meaning A secondary school class (16-17 year-olds) are working with trigonometry using the laws of sines and cosines. Before this episode the pupils have worked with the definitions and their application for calculating right-angled triangles. The 
teacher has presented the pupils with the laws of sines in the previous lesson and the pupils are asked to work in twos with the following task:

Make a sketch in GSP with a triangle, ABC. Measure the sides and the angles. Calculate the length of $a$ on the basis of $b$ and the angles $A$ and $B$. Move point $A$ around in a circle with the centre in $C$ and radius $b$. Prove that the $\sin A / \sin B$ relation is constant in all triangles thus produced.
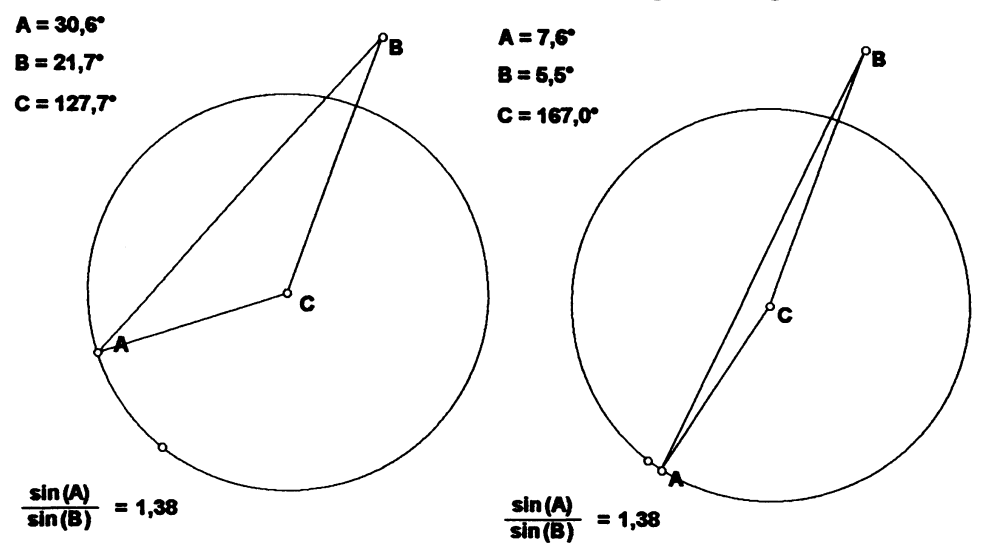

Figure 1: Two versions of the pupils' sketch with different positions of point A.

After approximately five minutes, two pupils have constructed a sketch which can be used to draw triangles with two constant sides (see figure 1). The pupils have made a formula in their sketch which calculates the desired relation and use the mouse to make point $A$ revolve quickly around the circle.

The pupils' dialogue was as follows (P1 is handling the mouse):

P1: Cool! It looks good and it's right! The relation is 1.38 the whole way round.

P2: OK, but we are supposed to prove it.

P1: Look at this. Even though the triangle is almost completely flat, it's still right.

( $A$ is placed so that the angle $C$ is almost $180^{\circ}$ ).

P2: Try over on the other side.

P1: What's $\sin \left(180^{\circ}\right)$ ? Isn't it 0 ? (P1 places $A$ so that the angle is almost $180^{\circ}$ ).

P2: Yes, $\sin (0)$ is too.

P1: But angle $B$ is not exactly 0.

P2: It's still strange that the relation is constant even though there's almost no triangle.

The teacher has observed that the pupils have used their sketch to investigate $\sin A / \sin B$ for different placing of $A$ and comes over to the pupils to see how their work is progressing. Here the notion that the relation is always 1.38 , regardless of where $A$ is placed, is used for the prompt from the teacher to see if they can produce a proof utilising references to earlier teaching and the work that 
they have done. In addressing this challenge, the first pupil then marks the line segments and the point $C$ and clicks on perpendicular in the construction menu. GSP then draws the line containing the height of $c$ in the triangle. P1 then revolves the point $A$ around the circle while P2 studies the proof on the blackboard from the previous lesson:

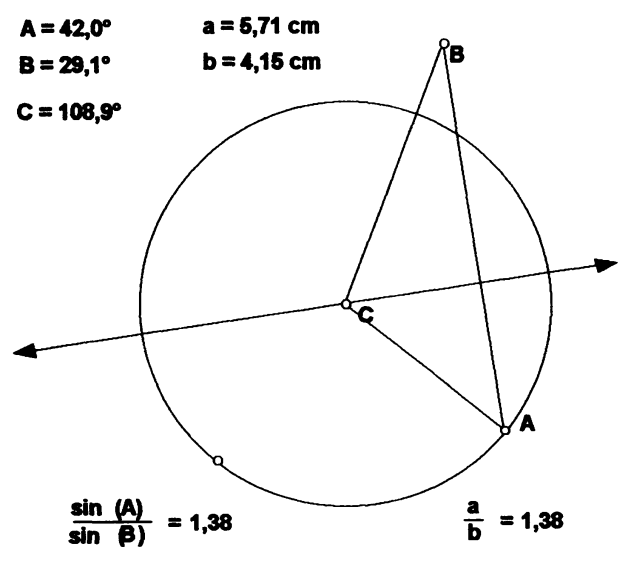
P1: If we call the height $h$ (meaning the height through $C$, see figure 2) we can use what's on the blackboard. (P2 writes down the formula from the board on paper while P1 watches.)
P2: Try calculating $a / b$ on the screen. (P1 marks the sides $a$ and $b$ in the triangle and clicks on length in the Measure menu, lengths of $a$ and $b$ appear, then P1 makes the formula $a / b$ using the Calculator menu in GSP and the formula appears (see figure 2).)

Figure 2 Shows the sketch the pupils made in connection with their proof.

P2: Look. that's right: 1.38, [P1 moves point $A$ in the circle.] $a$ and $b$ have the same length the whole time; therefore $a / b$ is constant and that's the same as $\sin A / \sin B$. We've proved it!

The pupils then call the teacher and explain how their approach uses that which appears on the board. The point is made that $a / b$ is constant, hence $\sin A / \sin B$ must also be constant and the teacher then engages in a dialogue which requires further pupil elaboration.

\section{Analysing the episode}

While the pupils are experimenting with the placement of point $A$ on the GSP figure, they notice in particular the 'flat' triangles that appear in the two extreme situations where angles $A$ and $C$, respectively, are close to $180^{\circ}$. They wonder intuitively at the fact that the relation $\sin A / \sin B$ can be constant under such extreme conditions and they may even be close to being able to formulate this wonder mathematically: How can a fraction where both numerator and denominator come close to 0 be constant? In this way the pupils' motivation 
changes in character. Now they actually wonder about it and they are concerned about finding a clarification.

What is characteristic in this episode is that it is the pupil's work with GSP that enables them to take up the teacher's challenge to make a proof. By working with GSP the pupils have created a personal meaning in their activity that is sufficiently in line with the intentions of the teacher. This in turn enables the teacher to engage in dialogue with the pupils, both supporting and challenging their work.

It is obvious that the use of GSP also functions as a filter when the teacher tries to discover what the pupils are doing and the learning of each individual pupil. A central question in this connection is the extent to which the pupils learn to interpret their GSP sketches as geometrical figures that establish certain relations between given geometrical objects and allow other relations to be free (Laborde, 1993). However, here it was quite clear that through utilising the possibilities for manipulation the pupils became aware of the decisive geometrical features of the figures they drew on the screen. It is, however, less clear whether the pupils actually experienced the fact that they had proved a consequence of the law of sines or if they were aware that the assertion followed as a simple algebraic consequence of the law of sines. In subsequent teaching, the teacher would need to try to clarify such questions-which, naturally, also arise in non-computer based teaching.

It is by now generally accepted in research on the didactics of mathematics that the creation of personal meaning in the teaching process is a decisive precondition for acquiring higher level mathematical understanding (e.g., see Steinbring, 1989).

\section{References}

Blomhøj, M. (1997). Computers in secondary mathematics teaching -a course on the law of sines and some didactical reflections (in Danish). In G. Dahland, (ed.) Elektroniska hjalpmedel $i$ matematikundervisningen. Institution for Pedagogic, Göteborgs University. Göteborgs University Press. 49-70.

Laborde, C. (1993). The Computer as part of the Learning Environment: The Case of Geometry. In C. Keitel and K. Ruthven (eds.) Learning from computers: Mathematics Education and Technology. Heidelberg: SpringerVerlag, 48-59.

Steinbring, H. (1989). Routine and meaning in the mathematics classroom. For the Learning of Mathematics, 9(1), 24-33. 